\title{
Feedback cooling of a single trapped ion
}

Pavel Bushev*, Daniel Rotter, Alex Wilson, François Dubin, Christoph Becher ${ }^{\S}$, Jürgen Eschner ${ }^{\dagger}$, and Rainer Blatt ${ }^{\ddagger}$ Institute for Experimental Physics, University of Innsbruck, Technikerstr. 25, A-6020 Innsbruck, Austria

Viktor Steixner ${ }^{\ddagger}$, Peter Rabl ${ }^{\ddagger}$, and Peter Zoller ${ }^{\ddagger}$

Institute for Theoretical Physics, University of Innsbruck, Technikerstr. 25, A-6020 Innsbruck, Austria

$\ddagger$ Institute for Quantum Optics and Quantum Information of the Austrian Academy of Sciences, 6020 Innsbruck, Austria

(Dated: October 29, 2018)

\begin{abstract}
Based on a real-time measurement of the motion of a single ion in a Paul trap, we demonstrate its electro-mechanical cooling below the Doppler limit by homodyne feedback control (cold damping). The feedback cooling results are well described by a model based on a quantum mechanical Master Equation.
\end{abstract}

PACS numbers: 3.65.Ta, 42.50.Lc 32.80.Pj, 42.50.Ct, 42.50.Vk, 32.80.Lg

Quantum optics, and more recently mesoscopic condensed matter physics, have taken a leading role in realizing individual quantum systems, which can be monitored continuously in quantum limited measurements, and at the same time can be controlled by external fields on time scales fast in comparison with the system evolution. Examples include cold trapped ions and atoms 1], cavity QED [2, 3, 4, 5] and nanomechanical systems [6]. This setting opens the possibility of manipulating individual quantum systems by feedback, a problem which is not only of a fundamental interest in quantum mechanics, but also promises a new route to generating interesting quantum states in the laboratory. First experimental efforts to realize quantum feedback have been reported only recently. While not all of them may qualify as quantum feedback in a strict sense, feedback has been applied to various quantum systems [5, 7, 8, 9, 10, 11]. On the theory side, this has motivated during the last decade the development of a quantum feedback theory [12, 13], where the basic ingredients are the interplay between quantum dynamics and the back-action of the measurement on the system evolution. In this letter we report a first experiment to demonstrate quantum feedback control, i.e. quantum feedback cooling, of a single trapped ion by monitoring the fluorescence of the laser driven ion in front of a mirror. We establish a continuous measurement of the position of the ion which allows us to act back in a feedback loop demonstrating "cold damping" 14, 15]. We will show that quantum control theory based on a quantum optical modelling of the system dynamics and continuous measurement theory of photodetection provides a quantitative understanding of the experimental results.

We study a single ${ }^{138} \mathrm{Ba}^{+}$ion in a miniature Paul trap which is continuously laser-excited and laser-cooled to the Doppler limit on its $\mathrm{S}_{1 / 2}$ to $\mathrm{P}_{1 / 2}$ transition at $493 \mathrm{~nm}$, as outlined in Fig. 1. The ion is driven by a laser near the atomic resonance, and the scattered light is emitted both into the radiation modes reflected by the mirror, as well as the other (background) modes of the quantized light field [16]. Light scattered into the mirror modes can either reach the photodetector directly, or after reflection from the mirror. From the resulting interference the motion of the ion (its projection onto the ion-mirror axis) is detected as a vibrational sideband in the fluctuation spectrum of the photon counting signal [17]. Of the three sidebands at about $(1,1.2,2.3) \mathrm{MHz}$, corresponding to the three axes of vibration, we observe the one at $\nu=1 \mathrm{MHz}$. It has a width $\Gamma \approx 400 \mathrm{~Hz}$ and is superimposed on the background shot noise generated by the photon counting process.

Our goal is to continuously read the position of the ion, and feed back a damping force proportional to the momentum to achieve feedback cooling. For a weakly driven atom the emitted light is dominantly elastic scattering at the laser frequency, and the information on the motion of

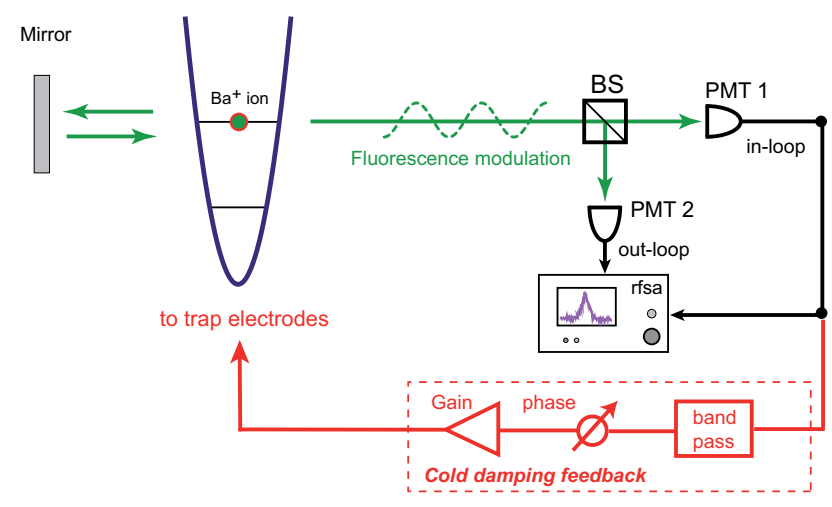

FIG. 1: A single ${ }^{138} \mathrm{Ba}^{+}$ion in a Paul trap (parabola) is laserexcited and -cooled on its $\mathrm{S}_{1 / 2}$ to $\mathrm{P}_{1 / 2}$ transition at $493 \mathrm{~nm}$. A retro-reflecting mirror $25 \mathrm{~cm}$ away from the trap and a lens (not shown) focus back the fluorescence onto the ion. The resulting interference fringes with up to $73 \%$ contrast are observed by a photomultiplier (PMT 1). The ion's oscillation in the trap creates an intensity modulation of the PMT signal which is observed as a sideband on a spectrum analyser (rfsa) 17]. For feedback cooling, the sideband signal is filtered, phase-shifted, and applied to the ion as a voltage on a trap electrode. 
the ion is encoded in the sidebands of the scattered light, displaced by the trap frequency $\nu$. For an ion trapped in the Lamb-Dicke regime $(\eta \sqrt{N} \ll 1$, with the Lamb Dicke parameter $\eta=2 \pi a_{0} / \lambda \sim 0.07, a_{0}$ the r.m.s. size of the trap ground state, $N$ the mean excitation number of the motional oscillator) the motional sidebands are suppressed by $\eta$ relative to the elastic component at the laser frequency. Thus the light reaching the detector will be the sum of the elastic component and weak sidebands [17], a situation reminiscent of homodyne detection, where a strong oscillator beats with the signal field to provide a homodyne current at the detector. This physical picture allows us to formulate the continuous readout of the position of the ion as well as the quantum feedback cooling in the well-developed language of homodyne detection and quantum feedback.

The homodyne current at the photodetector (see Fig. 1) with the (large) signal from the elastic light scattering subtracted has the form

$$
I_{c}(t)=\gamma \eta\langle\hat{z}\rangle_{c}(t)+\sqrt{\frac{\gamma}{2}} \xi(t) .
$$

The first term is proportional to the conditioned expectation value of the position of the trapped ion, $\langle\hat{z}\rangle_{c}(t)$, and the second term is a shot noise contribution with Gaussian white noise $\xi(t)$. We have defined $\hat{z}=a+a^{\dagger} \equiv z / a_{0}$ with $a\left(a^{\dagger}\right)$ destruction (creation) operator of the harmonic oscillator, and we have assumed that the trap center is placed at a distance $L=n \lambda / 2+\lambda / 8$ ( $n$ integer) from the mirror, corresponding to a point of maximum slope of the standing wave intensity of the mirror mode. The current $I_{c}(t)$ scales with $\gamma \propto \epsilon$, which is the light scattering rate into the solid angle $(4 \pi \epsilon)$ of the mirror mode induced by the laser. The expectation value $\langle\cdot\rangle_{c} \equiv \operatorname{Tr}\left\{\cdot \rho_{c}(t)\right\}$ is defined with respect to a conditioned density operator $\rho_{c}(t)$, which reflects our knowledge of the motional state of the ion for the given history of the photocurrent. According to the theory of homodyne detection, $\rho_{c}(t)$ obeys the Ito stochastic differential equation [18]

$$
\begin{aligned}
d \rho_{c}(t)= & -\mathrm{i} \nu\left[a^{\dagger} a, \rho_{c}(t)\right] d t+\mathcal{L}_{0} \rho_{c}(t) d t \\
& +\sqrt{2 \gamma \eta^{2}} \mathcal{H} \rho_{c}(t) d W(t),
\end{aligned}
$$

where $\mathcal{H} \rho_{c}(t)=\left(\hat{z} \rho_{c}(t)+\rho_{c}(t) \hat{z}-2\langle\hat{z}\rangle_{c}(t) \rho_{c}(t)\right)$. The first line determines the unobserved evolution of the ion, including the harmonic motion in the trap with frequency $\nu$ and the dissipative dynamics, $\mathcal{L}_{0}$, due to photon scattering. The latter is given by

$$
\mathcal{L}_{0} \rho=\Gamma(N+1) \mathcal{D}[a] \rho+\Gamma N \mathcal{D}\left[a^{\dagger}\right] \rho,
$$

where we have defined the superoperator $\mathcal{D}[c] \rho \equiv c \rho c^{\dagger}-$ $\left(c^{\dagger} c \rho+\rho c^{\dagger} c\right) / 2$. The laser cooling rate $\Gamma$ and the steady state occupation number, $N=\left\langle a^{\dagger} a\right\rangle$, can either be estimated from the motional sidebands or deduced from the cooling laser parameters [1]. In the present experiment, $N \approx 17$ corresponds to the Doppler limit. The last term of Eq. (2) is proportional to the Wiener increment $d W(t) \equiv \xi(t) d t$ and corresponds to an update of the observers knowledge about the system according to a certain measurement result $I_{c}(t)$. In summary, Eq. (1) demonstrates that observation of the sidebands of the light scattered into the mirror mode provides us with information of the position of the ion, while the system density matrix is updated according to Eq. (2). This is the basis for describing feedback control of the ion, as shown in the following.

For feedback cooling, the vibrational sideband is extracted with a bandpass filter of bandwidth $B=30 \mathrm{kHz}$, shifted by $(-\pi / 2)$, amplified, and the resulting output voltage is applied to an electrode which is close to the trap inside the vacuum. Thereby we create a driving force which is proportional to (and opposed to) the instantaneous velocity of the ion and which thus adds to the damping of its vibration. The overall gain of the feedback loop depends on many factors such as the fringe contrast of the interference, the PMT characteristics, etc. It is varied electronically by setting the amplification $G$ of the final amplifier in the loop. We can also set the phase to other values than the optimum of $(-\pi / 2)$, which will be used in order to compare experiment and theory.

To analyze the result of the feedback we look at the changes in the sideband spectrum. The modified spectra require careful interpretation. The spectrum observed inside the feedback loop ("in-loop" or PMT1 in Fig. 1) shows not only the motion of the ion, but the sum of the motion and the shot noise. As the feedback correlates these fluctuations, a reduction of the signal below the shot noise level may occur, similar in appearance to signatures of squeezed light. This effect is known as "squashing" 19], or "anti-correlated state of light" in an opto-electronic loop [20], and it does not constitute a quantum mechanical squeezing of the fluctuations 21, 22]. The effect on the motion can only be reliably detected by splitting the optical signal before it is measured and recording it outside the feedback loop with a second PMT ("out-loop" in Fig. 1), whose shot noise is not correlated with the motion.

In Fig. 2 we show spectra recorded with the spectrum analyzer, and measured outside and inside the feedback loop. The first curve of each row, showing the largest sideband, is the one without feedback (gain $G=0$ ). The other two curves are recorded with the loop closed (gain values $G=1.3$ and 8.7). The sub shot noise fluctuations inside the loop, when the ion is driven to move in antiphase with the shot noise, are clearly visible in Fig. 2(f). The main cooling results are curves (b) and (c) which show the motional sideband reduced in size and broadened, indicating a reduced energy (proportional to the area under the curve) and a higher damping rate (the width). From case (b) to (c) the area increases, as the injected and amplified shot noise overcompensates the increased damping. As shown below, in our model the 

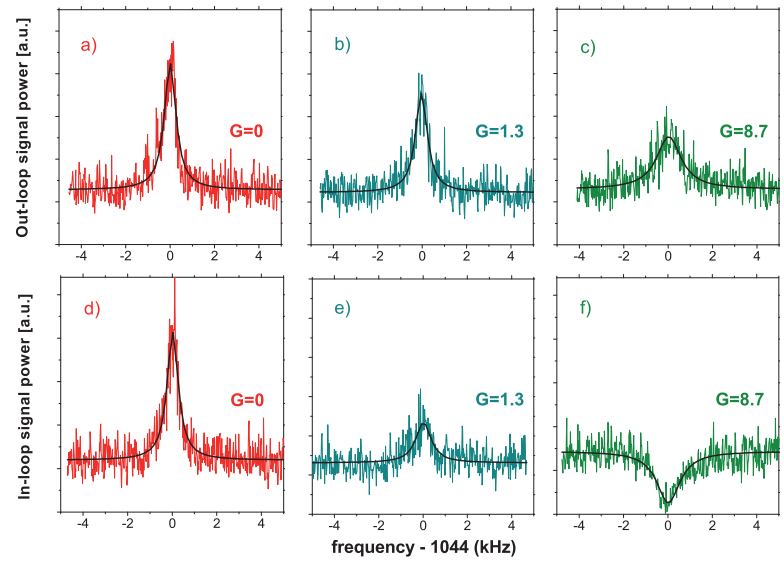

FIG. 2: Feedback cooling spectra. The vibrational sideband around $\nu=1 \mathrm{MHz}$ is shown on top of the spectrally flat shot noise background, to which the spectra are normalized. The upper curves (a)-(c) are measured outside the feedback loop, while the lower curves (d)-(f) are the in-loop results. Spectra (a) and (d) are for laser cooling only, the other curves are recorded with feedback at the indicated gain values. The feedback phase is set to $(-\pi / 2)$. The gain values indicate the settings of the final amplifier in the feedback loop.

incorporation of quantum feedback competing with laser cooling predicts such behavior, i.e., the existence of an optimal gain for maximal cooling (for a detailed description cf. [23]).

We model the effect of the feedback force acting on the ion by extending Eq. (2) with the feedback contribution,

$$
\left[d \rho_{c}(t)\right]_{\mathrm{fb}}=-\mathrm{i} \tilde{G} I_{\mathrm{fb}, c}(t-\tau)\left[\hat{z}, \rho_{c}(t)\right],
$$

where $I_{\mathrm{fb}, c}(t)$ denotes the measured current after the feedback circuit. The latter is proportional to the voltage applied to the trap electrodes. All conversion factors between the feedback current and the actual force applied on the ion are included in the overall gain $\tilde{G}$. The time delay $\tau$ in the feedback loop preserves causality and is small compared to the fastest timescale $\nu^{-1}$ of the motion of the ion which allows us to consider the Markovian limit $\left(\tau \rightarrow 0^{+}\right)$.

To obtain an expression for the feedback current $I_{\mathrm{fb}, c}(t)$, we change into a frame rotating with the trap frequency $\nu$ and define the density operator, $\mu_{c}(t) \equiv$ $\exp \left(\mathrm{i} \nu a^{\dagger} a t\right) \rho_{c}(t) \exp \left(-\mathrm{i} \nu a^{\dagger} a t\right)$, evolving on the (slow) cooling timescales. This is convenient due to the large separation between the timescale of the harmonic oscillations, $\nu^{-1}$ and the timescale of laser and feedback cooling in our experiment. For our experimental parameters, $\nu \gg B \gg \Gamma$, the feedback current for a phase shift of $(-\pi / 2)$ has the form 23]

$$
I_{\mathrm{fb}, c}(t)=\left(\gamma \eta\langle\hat{p}\rangle_{c}(t)+\sqrt{\frac{\gamma}{2}} \Xi(t)\right) \cos (\nu t),
$$

where $\langle\hat{p}\rangle_{c}(t) \equiv \operatorname{Tr}\left\{\hat{p} \mu_{c}(t)\right\}$, and $\hat{p} \equiv \mathrm{i}\left(a^{\dagger}-a\right)$ is the momentum operator conjugated to $\hat{z}$. The first term in this current therefore provides damping for the motion of the ion. The second term of Eq. (5) describes the shot noise which passes through the electronic circuit and is fed back to the ion. The stochastic variable $\Xi(t)$ is Gaussian white noise on a timescale given by the inverse bandwidth $B^{-1}$, whereby $B \gg \Gamma$ implies that it is spectrally flat on the frequency range of the cooling dynamics.

For a full record of the photocurrent $I_{c}(t)$, Eqs. (2) and (4) determine the evolution of the ion's motional state in presence of feedback. As it is impractical to keep track of the whole photocurrent in the experiment, we derive a master equation for the density operator averaged over all possible realisation of $I_{c}(t), \mu(t)$. Along the ways of the Wiseman-Milburn theory of quantum feedback 12], for a phase shift of $(-\pi / 2)$, we obtain the quantum feedback master equation 23]

$$
\dot{\mu}=\mathcal{L}_{0} \mu-\mathrm{i} \frac{\tilde{G} \gamma \eta}{4}[\hat{z}, \hat{p} \mu+\mu \hat{p}]-\frac{\tilde{G}^{2} \gamma}{16}[\hat{z},[\hat{z}, \mu]] .
$$

The second and third term are the additional contributions due to the feedback. The part linear in $\tilde{G}$ induces damping of the motion of the ion, and the term quadratic in $\tilde{G}$ describes the effect of the fed back noise leading to diffusion of the momentum. The competition between laser cooling, damping and the injected noise leads to the characteristic behavior of the steady state number expectation value

$$
\langle n\rangle_{s s}=\frac{N+\eta \gamma \tilde{G}(2 N-1) / 2 \Gamma+\gamma \tilde{G}^{2} / 8 \Gamma}{1+2 \eta \gamma \tilde{G} / \Gamma} .
$$

For small gain, damping dominates, and the energy of the ion is decreased below the Doppler limit. For higher gain, the diffusive term describing the noise fed back into the system overcompensates cooling, i.e., heats the ion. Consequently, for $(-\pi / 2)$ feedback phase and optimal gain conditions the steady state energy is minimized. On the contrary, for a $\pi$ phase shift $(-\hat{z})$ replaces $\hat{p}$ in the second term of Eq. (6), the feedback force then merely induces a frequency shift, $\Delta \omega=\tilde{G} \gamma \eta / 2$, but no damping. Increasing the gain then always enhances the steady state number expectation value, i.e., the mean ion energy. We now compare the theoretical predictions and the experimental results for the feedback phases of physical interest, namely $(-\pi / 2)$ and $\pi$.

The measured ion energy as a function of the feedback electronic gain is shown in Fig. 3. On the first side, as expected, for a $(-\pi / 2)$ feedback phase, cooling by more than $30 \%$ below the Doppler limit is achieved, while further increase of the gain drives the shot noise and therefore heats the motion of the ion. On the other side, a $\pi$ phase shift in the feedback loop does not yield any damping, in such conditions the motion of the ion is 


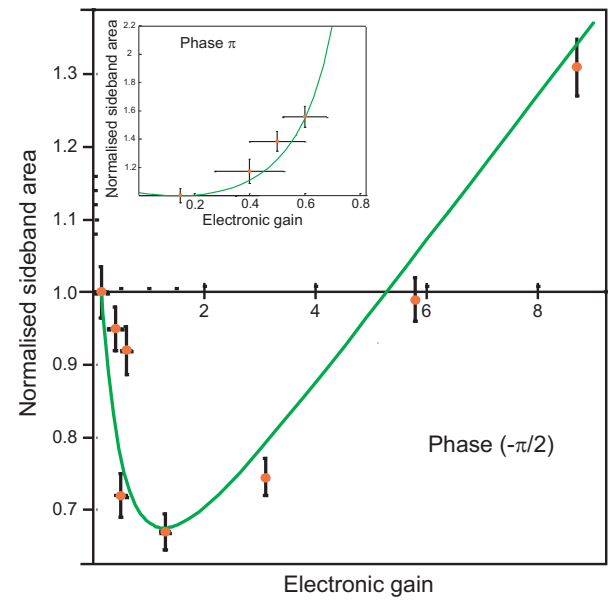

FIG. 3: Steady-state energy of the cooled oscillator: measured sideband area, normalized to the value without feedback, versus gain of the feedback loop, for $(-\pi / 2)$ (a) and $\pi$ (b) feedback phase. The curves are the model calculations. The gain axis is scaled to the experimental values of the electronic gain.

only driven. This results in an increase of the measured sideband area (as shown in the insert of Figure 3), as well as a shift of the sideband center frequency (graph not shown). Both cases demonstrate good agreement between experiment and theory. Finally, let us stress that the optimal cooling rate is governed by the collection efficiency of the fluorescence going into the mirror mode, $\epsilon$. In the experiments presented above, $\epsilon \approx 1 \%$ leads to a decrease of the steady state occupation number $N$ from 17 to 12 , while $N \approx 3$ can be reached for $\epsilon \approx 15 \%$, experimentally achievable for optimal optical coupling.

To summarize, we have demonstrated real-time feedback cooling of the motion of a single trapped ion. Electro-mechanical back-action based on a sensitive realtime measurement of the motion of the ion in the trap allowed us to cool one motional degree of freedom by $30 \%$ below the Doppler limit. Unlike with laser cooling, the presented method allows to cool one of the ion's motional mode without heating the two others, and our procedure can easily be extended to cooling all motional modes. The cooling process is shot-noise limited and the fraction of scattered photons recorded to observe the motion of the ion limits the ultimate cooling at optimum gain. The latter can yield a steady state occupation number $N=3$ for realistic experimental conditions. In conclusion, our feedback scheme offers a possible way to very efficiently cool the motion of ions unsuitable for sideband cooling.

Acknowledgements. This work has been supported by the Austrian Science Fund (FWF) in the project SFB15, by the European Commission (QUEST network, HPRNCT-2000-00121, QUBITS network, IST1999-13021), and by the "Institut für Quanteninformation GmbH". We thank S. Mancini, A. Masalov, and D.
Vitali for clarifying discussions. P. B. thanks the group of theoretical physics at U. Camerino, Italy, for hospitality.

[*] Present address: Laboratory of Physical Chemistry, ETH Zürich, Switzerland.

[§] Present address: Fachrichtung Technische Physik, Saarland University, Saarbrücken, Germany.

$\left[{ }^{\dagger}\right]$ Present address: ICFO - Institut de Ciències Fotòniques, Barcelona, Spain.

[1] D. Leibfried, R. Blatt, C. Monroe, and D. Wineland, Rev. Mod. Phys 75, 281 (2003), and references cited.

[2] M. Keller, B. Lange, K. Hayasaka, W. Lange, H. Walther, Nature 431, 1075 (2004).

[3] J. McKeever, A. Boca, A. D. Boozer, R. Miller, J. R. Buck, A. Kuzmich, and H. J. Kimble, Science 303, 1992 (2004); P. Maunz, T. Puppe, I. Schuster, N. Syassen, P. W. H. Pinkse, and G. Rempe, Nature 428, 50 (2004)

[4] T. Legero, T. Wilk, M. Hennrich, G. Rempe, and A. Kuhn, Phys. Rev. Lett. 93, 070503 (2004).

[5] J. E. Reiner, W. P. Smith, L. A. Orozco, H. M. Wiseman, and Jay Gambetta, Phys. Rev. A. 70, 023819 (2004).

[6] A. Hopkins, K. Jacobs, S. Habib, and K. Schwab, Phys. Rev. B 68, 235328 (2003)

[7] D. A. Steck, K. Jacobs, H. Mabuchi, T. Bhattacharya, and S. Habib, Phys. Rev. Lett 92, 223004 (2004).

[8] B. D'Urso, B. Odom, and G. Gabrielse, Phys. Rev. Lett. 90, 043001 (2003).

[9] T. Fischer, P. Maunz, P. W. H. Pinkse, T. Puppe, and G. Rempe, Phys. Rev. Lett. 88, 163002 (2002).

[10] N. V. Morrow, S. K. Dutta, and G. Reithel, Phys. Rev. Lett. 88, 093003 (2002).

[11] J.M. Geremia, J. K. Stockton, H. Mabuchi, Science 304, 270 (2004); D. Oblak, P. G. Petrov, C. L. Garrido Alzar, W. Tittel, A. K. Vershovski, J. K. Mikkelsen, J. L. Sørensen, and E. S. Polzik, Phys. Rev. A 71, 43807 (2005).

[12] H.M. Wiseman and G. J. Milburn, Phys. Rev. Lett. 70, 548-551 (1993).

[13] S. Mancini, D. Vitali, and P. Tombesi, Phys. Rev. Lett. 80, 688-691 (1998); D. Vitali, S. Mancini, L. Ribichini, and P. Tombesi, Phys. Rev. A 65, 063803 (2002).

[14] J. M. W. Milatz and J. J. Van Zolingen, Physica XIX, 181(1953).

[15] P. F. Cohadon, A. Heidmann, and M. Pinard, Phys. Rev. Lett. 83, 3174 (1999).

[16] U. Dorner and P. Zoller Phys. Rev. A 66, 023816 (2002).

[17] P. Bushev, A. Wilson, J. Eschner, C. Raab, F. SchmidtKaler, C. Becher, and R. Blatt, Phys. Rev. Lett. 92, 223602 (2004); J. Eschner, Ch. Raab, F. Schmidt-Kaler, and R. Blatt, Nature 413, 495-498 (2001).

[18] C. W. Gardiner and P. Zoller, Quantum Noise (Springer, Berlin, 2004), and references cited.

[19] B. C. Buchler, M. B. Gray, D. A. Shaddock, T. C. Ralph, D. E. McClelland, Opt. Lett. 24, 259 (1999).

[20] A. V. Masalov, A. A. Putilin, and M. V. Vasilyev, J. Mod. Optics 41, 1941 (1994).

[21] H. A. Haus, Y. Yamamoto, Phys. Rev. A 34, 270-292 (1986).

[22] J. H. Shapiro, G. Saplakoglu, S.-T. Ho, P. Kumar, B. E. A. Saleh, M. C. Teich, J. Opt. Soc. Am. B 4, 1604-1620 
(1987).

[23] V. Steixner, P. Rabl, and P. Zoller, accepted by Phys.
Rev. A, quant-ph/0506187 